\title{
Effect of Hospital Readmission Reduction on Patients at Low, Medium, and High Risk of Readmission in the Medicare Population
}

\author{
Saul Blecker, MD, MHS ${ }^{1,2,3}$, Jeph Herrin, PhD,,5, Ji Young Kwon, MPH ${ }^{6}$, Jacqueline N. Grady, MS \\ Simon Jones, $\mathrm{PhD}^{1,3}$, Leora I. Horwitz, MD, $\mathrm{MHS}^{1,2,3}$
}

\begin{abstract}
${ }^{1}$ Division of Healthcare Delivery Science, Department of Population Health, NYU School of Medicine, New York, New York; ${ }^{2}$ Division of General Internal Medicine and Clinical Innovation, Department of Medicine, NYU School of Medicine, New York, New York; ${ }^{3}$ Center for Healthcare Innovation and Delivery Science, NYU Langone Medical Center, New York, New York; ${ }^{4}$ Section of Cardiovascular Medicine, Yale School of Medicine, New Haven, Connecticut; ${ }^{5} \mathrm{Health}$ Research \& Educational Trust, Chicago, Illinois; ${ }^{\circ} \mathrm{Center}$ for Outcomes Research and Evaluation, Yale-New Haven Hospital, New Haven, Connecticut.
\end{abstract}

BACKGROUND: Hospitalization and readmission rates have decreased in recent years, with the possible consequence that hospitals are increasingly filled with high-risk patients.

OBJECTIVE: We studied whether readmission reduction has affected the risk profile of hospitalized patients and whether readmission reduction was similarly realized among hospitalizations with low, medium, and high risk of readmissions.

DESIGN: Retrospective study of hospitalizations between January 2009 and June 2015.

PATIENTS: Hospitalized fee-for-service Medicare beneficiaries, categorized into 1 of 5 specialty cohorts used for the publicly reported hospital-wide readmission measure.

MEASUREMENTS: Each hospitalization was assigned a predicted risk of 30-day, unplanned readmission using a risk-adjusted model similar to publicly reported measures. Trends in monthly mean predicted risk for each cohort and trends in monthly observed to expected readmission for hospitalizations in the lowest $20 \%$, middle $60 \%$, and highest $20 \%$ of risk of readmission were assessed using time series models.

RESULTS: Of 47,288,961 hospitalizations, $16.2 \%$ ( $n=$ $7,642,161$ ) were followed by an unplanned readmission within 30 days. We found that predicted risk of readmission increased by $0.24 \%(P=.03)$ and $0.13 \%$ $(P=.004)$ per year for hospitalizations in the surgery/ gynecology and neurology cohorts, respectively. We found no significant increase in predicted risk for hospitalizations in the medicine $(0.12 \%, P=.12)$, cardiovascular $(0.32 \%$, $P=.07)$, or cardiorespiratory $(0.03 \%, P=.55)$ cohorts. In each cohort, observed to expected readmission rates steadily declined, and at similar rates for patients at low, medium, and high risk of readmission.

CONCLUSIONS: Hospitals have been effective at reducing readmissions across a range of patient risk strata and clinical conditions. The risk of readmission for hospitalized patients has increased for 2 of 5 clinical cohorts. Journal of Hospital Medicine 2018;13:537-543. Published online first February 12, 2018. (C) 2018 Society of Hospital Medicine

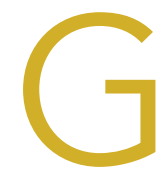

iven the high cost of readmissions to the healthcare system, there has been a substantial push to reduce readmissions by policymakers. ${ }^{1}$ Among these is the Hospital Readmissions Reduction Program (HRRP), in which hospitals with higher than expected readmission rates receive reduced payments from Medicare. ${ }^{2}$ Recent evidence has suggested the success of such policy changes, with multiple reports demonstrating a decrease in 30-day re-

*Address for correspondence: Saul Blecker, MD, MHS, NYU School of Medicine, 227 E. 30th St., Room 734, New York, NY 10016; Telephone: 646-501-2513; Fax: 646-501-2706; E-mail: Saul.blecker@nyumc.org

Additional Supporting Information may be found in the online version of this article.

Received: July 25, 2017; Revised: November 10, 2017; Accepted: November 22, 2017

๑ 2018 Society of Hospital Medicine DOI 10.12788/jhm.2936 admission rates in the Medicare population starting in 2010..$^{3-8}$

Initiatives to reduce readmissions can also have an effect on total number of admissions.., 10 Indeed, along with the recent reduction in readmission, there has been a reduction in all admissions among Medicare beneficiaries. ${ }^{11,12}$ Some studies have found that as admissions have decreased, the burden of comorbidity has increased among hospitalized patients, 3,11 suggesting that hospitals may be increasingly filled with patients at high risk of readmission. However, whether readmission risk among hospitalized patients has changed remains unknown, and understanding changes in risk profile could help inform which patients to target with future interventions to reduce readmissions.

Hospital efforts to reduce readmissions may have differential effects on types of patients by risk. For instance, low-intensity, system-wide interventions such as standardized discharge instructions or medicine reconciliation may have a stronger effect on patients at relatively low risk of readmission who may have a 
few important drivers of readmission that are easily overcome. Alternatively, the impact of intensive care transitions management might be greatest for high-risk patients, who have the most need for postdischarge medications, follow-up, and selfcare.

The purpose of this study was therefore twofold: (1) to observe changes in average monthly risk of readmission among hospitalized Medicare patients and (2) to examine changes in readmission rates for Medicare patients at various risk of readmission. We hypothesized that readmission risk in the Medicare population would increase in recent years, as overall number of admissions and readmissions have fallen.7,11 Additionally, we hypothesized that standardized readmission rates would decline less in highest risk patients as compared with the lowest risk patients because transitional care interventions may not be able to mitigate the large burden of comorbidity and social issues present in many high-risk patients. ${ }^{13,14}$

\section{METHODS}

We performed a retrospective cohort study of hospitalizations to United States nonfederal short-term acute care facilities by Medicare beneficiaries between January 2009 and June 2015. The design involved four steps. First, we estimated a predictive model for unplanned readmissions within 30 days of discharge. Second, we assigned each hospitalization a predicted risk of readmission based on the model. Third, we studied trends in mean predicted risk of readmission during the study period. Fourth, we examined trends in observed to expected (O/E) readmission for hospitalizations in the lowest, middle, and highest categories of predicted risk of readmission to determine whether reductions in readmissions were more substantial in certain risk groups than in others.

Data were obtained from the Centers for Medicare and Medicaid Services (CMS) Inpatient Standard Analytic File and the Medicare Enrollment Data Base. We included hospitalizations of fee-for-service Medicare beneficiaries age $\geq 65$ with continuous enrollment in Part A Medicare fee-for-service for at least one year prior and 30 days after the hospitalization. ${ }^{15}$ Hospitalizations with a discharge disposition of death, transfer to another acute hospital, and left against medical advice (AMA) were excluded. We also excluded patients with enrollment in hospice care prior to hospitalization. We excluded hospitalizations in June 2012 because of an irregularity in data availability for that month.

Hospitalizations were categorized into five specialty cohorts according to service line. The five cohorts were those used for the CMS hospital-wide readmission measure and included surgery/gynecology, medicine, cardiovascular, cardiorespiratory, and neurology. ${ }^{15}$ Among the three clinical conditions tracked as part of HRRP, heart failure and pneumonia were a subset of the cardiorespiratory cohort, while acute myocardial infarction was a subset of the cardiovascular cohort. Our use of cohorts was threefold: first, the average risk of readmission differs substantially across these cohorts, so pooling them produces heterogeneous risk strata; second, risk variables perform differently in different cohorts, so one single model may not be as accurate for calculating risk; and, third, the use of disease cohorts makes our results comparable to the CMS model and similar to other readmission studies in Medicare. ${ }^{7,8,15}$

For development of the risk model, the outcome was 30-day unplanned hospital readmission. Planned readmissions were excluded; these were defined by the CMS algorithm as readmissions in which a typically planned procedure occurred in a hospitalization with a nonacute principal diagnosis. ${ }^{16}$ Independent variables included age and comorbidities in the final hospital-wide readmission models for each of the five specialty cohorts. ${ }^{15}$ In order to produce the best possible individual risk prediction for each patient, we added additional independent variables that CMS avoids for hospital quality measurement purposes but that contribute to risk of readmission: sex, race, dual eligibility status, number of prior AMA discharges, intensive care unit stay during current hospitalization, coronary care unit stay during current hospitalization, and hospitalization in the prior 30, 90, and 180 days. We also included an indicator variable for hospitalizations with more than 9 discharge diagnosis codes on or after January 2011, the time at which Medicare allowed an increase of the number of International Classification of Diseases, 9th Revision-Clinical Modification diagnosis billing codes from 9 to $25 .{ }^{17}$ This indicator adjusts for the increased availability of comorbidity codes, which might otherwise inflate the predicted risk relative to hospitalizations prior to that date.

Based on the risk models, each hospitalization was assigned a predicted risk of readmission. For each specialty cohort, we pooled all hospitalizations across all study years and divided them into risk quintiles. We categorized hospitalizations as high risk if in the highest quintile, medium risk if in the middle three quintiles, and low risk if in the lowest quintile of predicted risk for all study hospitalizations in a given specialty cohort.

For our time trend analyses, we studied two outcomes: monthly mean predicted risk and monthly ratio of observed readmissions to expected readmissions for patients in the lowest, middle, and highest categories of predicted risk of readmission. We studied monthly predicted risk to determine whether the average readmission risk of patients was changing over time as admission and readmission rates were declining. We studied the ratio of $O / E$ readmissions to determine whether the decline in overall readmissions was more substantial in particular risk strata; we used the ratio of O/E readmissions, which measures number of readmissions divided by number of readmissions predicted by the model, rather than crude observed readmissions, as O/E readmissions account for any changes in risk profiles over time within each risk stratum. Independent variables in our trend analyses were year - entered as a continuous variable - and indicators for postintroduction of the Affordable Care Act (ACA, March 2010) and for postintroduction of HRRP (October 2012); these time indicators were included because of prior studies demonstrating that the introduction of ACA was associated with a decrease from baseline in readmission rates, which leveled off after introduction of HRRP. ${ }^{7}$ We also included an indicator for calendar quarter to account for seasonal effects. 
Statistical Analysis

We developed generalized estimating equation models to predict 30-day unplanned readmission for each of the five specialty cohorts. The five models were fit using all patients in each cohort for the included time period and were adjusted for clustering by hospital. We assessed discrimination by calculating area under the receiver operating characteristic curve (AUC) for the five models; the AUCs measured the models' ability to distinguish patients who were readmitted versus those who were not. ${ }^{18}$ We also calculated AUCs for each year to examine model performance over time.

Using these models, we calculated predicted risk for each hospitalization and averaged these to obtain mean predicted risk for each specialty cohort for each month. To test for trends in mean risk, we estimated 5 time series models, one for each cohort, with the dependent variable of monthly mean predicted risk. For each cohort, we first estimated a series of 12 empty autoregressive models, each with a different autoregressive term $(1,2 \ldots 12)$. For each model, we calculated $\chi^{2}$ for the test that the autocorrelation was 0 ; based on a comparison of chisquared values, we specified an autocorrelation of 1 month for all models. Accordingly, a one-month lag was used to estimate one final model for each cohort. Independent variables included year and indicators for post-ACA and post-HRRP; these variables captured the effect of trends over time and the introduction of these policy changes, respectively. ${ }^{19}$

To determine whether changes in risk over time were a result of changes in particular risk groups, we categorized hospitalizations into risk strata based on quintiles of predicted risk for each specialty cohort for the entire study period. For each individual year, we calculated the proportion of hospitalizations in the highest, middle, and lowest readmission risk strata for each cohort.

We calculated the monthly ratio of O/E readmission for hospitalizations in the lowest $20 \%$, middle $60 \%$, and highest $20 \%$ of readmission risk by month; O/E reflects the excess or deficit observed events relative to the number predicted by the model. Using this monthly $\mathrm{O} / \mathrm{E}$ as the dependent variable, we developed autoregressive time series models as above, again with a one-month lag, for each of these 3 risk strata in each cohort. As before, independent variables were year as a continuous variable, indicator variables for post-ACA and post-HRRP, and a categorical variable for calendar quarter.

All analyses were done in SAS version 9.3 (SAS Institute Inc., Cary, North Carolina) and Stata version 14.2 (StataCorp LLC, College Station, Texas).

\section{RESULTS}

We included $47,288,961$ hospitalizations in the study, of which $11,231,242(23.8 \%)$ were in the surgery/gynecology cohort, $19,548,711$ (41.3\%) were in the medicine cohort, 5,433,125 (11.5\%) were in the cardiovascular cohort, 8,179,691 (17.3\%) were in the cardiorespiratory cohort, and 2,896,192 (6.1\%) were in the neurology cohort. The readmission rate was $16.2 \%(n=$ $7,642,161)$ overall, with the highest rates observed in the cardiorespiratory $(20.5 \%)$ and medicine $(17.6 \%)$ cohorts and the

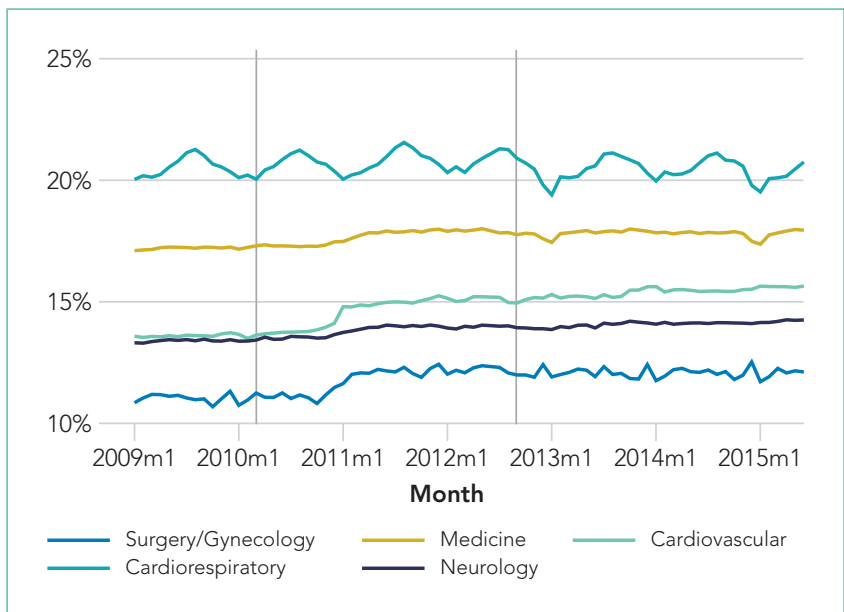

FIG 1. Trends in monthly mean predicted risk of readmission, by specialty cohort. Vertical lines represent introduction of the Affordable Care Act in March 2010 and introduction of the Hospital Readmissions Reduction Program in October 2012.

lowest rates observed in the surgery/gynecology (11.8\%) and neurology (13.8\%) cohorts.

The final predictive models for each cohort ranged in number of parameters from 56 for the cardiorespiratory cohort to 264 for the surgery/gynecology cohort. The models had AUCs of $0.70,0.65,0.67,0.65$, and 0.63 for the surgery/gynecology, medicine, cardiovascular, cardiorespiratory, and neurology cohorts, respectively; AUCs remained fairly stable over time for all disease cohorts (Appendix Table 1).

We observed an increase in the mean predicted readmission risk for hospitalizations in the surgery/gynecology and cardiovascular hospitalizations in early 2011 (Figure 1), a period between the introduction of ACA in March 2010 and the introduction of HRRP in October 2012. In time series models, the surgery/gynecology, cardiovascular, and neurology cohorts had increased predictive risks of readmission of $0.24 \%$, $0.32 \%$, and $0.13 \%$ per year, respectively, although this difference did not reach statistical significance for the cardiovascular cohort (Table 1). We found no association between introduction of ACA or HRRP and predicted risk for these cohorts (Table 1). There were no trends or differences in predicted readmission risk for hospitalizations in the medicine cohort. We observed a seasonal variation in predicted readmission risk for the cardiorespiratory cohort but no notable change in predicted risk over time (Figure 1); in the time series model, there was a slight decrease in risk following introduction of HRRP (Table 1).

After categorizing hospitalizations by predicted readmission risk, trends in the percent of hospitalizations in low, middle, and high risk strata differed by cohort. In the surgery/gynecology cohort, the proportion of hospitalizations in the lowest risk stratum increased only slightly, from $20.1 \%$ in 2009 to $21.1 \%$ of all surgery/gynecology hospitalizations in 2015 (Appendix Table 2). The proportion of surgery/gynecology hospitalizations in the high risk stratum (top quintile of risk) increased from $16.1 \%$ to $21.6 \%$ between 2009 and 2011 and remained 
TABLE 1. Beta Coefficients from the Times Series Models Examining the Association Between Time and Predicted Risk of Readmission Following Hospitalization

\begin{tabular}{|c|c|c|c|c|c|c|c|c|c|c|}
\hline \multirow[b]{2}{*}{$\begin{array}{l}\text { Model } \\
\text { Variable }\end{array}$} & \multicolumn{2}{|c|}{ Surgery/Gynecology } & \multicolumn{2}{|c|}{ Medicine } & \multicolumn{2}{|c|}{ Cardiovascular } & \multicolumn{2}{|c|}{ Cardiopulmonary } & \multicolumn{2}{|c|}{ Neurology } \\
\hline & $\begin{array}{l}\text { Beta Coefficient } \\
\text { (SE) }\end{array}$ & $P$ Value & $\begin{array}{l}\text { Beta Coefficient } \\
\text { (SE) }\end{array}$ & $P$ Value & $\begin{array}{l}\text { Beta Coefficient } \\
\text { (SE) }\end{array}$ & $P$ Value & $\begin{array}{l}\text { Beta Coefficient } \\
\text { (SE) }\end{array}$ & $P$ Value & $\begin{array}{l}\text { Beta Coefficient } \\
\text { (SE) }\end{array}$ & $P$ Value \\
\hline Time (years) & $0.236(0.109)$ & .03 & $0.118(0.076)$ & .12 & $0.317(0.171)$ & .065 & $0.027(0.044)$ & .547 & $0.131(0.045)$ & .004 \\
\hline post-ACA & $-0.108(0.237)$ & .649 & $0.006(0.202)$ & .976 & $0.103(0.224)$ & .646 & $0.116(0.169)$ & .491 & $0.103(0.100)$ & .302 \\
\hline post-HRRP & $-0.125(0.361)$ & .729 & $-0.008(0.194)$ & .967 & $0.090(0.447)$ & .841 & $-0.319(0.153)$ & .038 & $-0.018(0.122)$ & .883 \\
\hline Intercept & $10.963(0.322)$ & $<.001$ & $17.196(0.284)$ & $<.001$ & $13.639(0.608)$ & $<.001$ & $20.142(0.168)$ & $<.001$ & $13.326(0.128)$ & $<.001$ \\
\hline
\end{tabular}

NOTE: Years represents change with each year; post-ACA represents change with introduction of the ACA in March 2010; post-HRRP represents change with introduction of the HRRP in October 2012. Adjusted for calendar quarter. Abbreviations: ACA, Affordable Care Act; HRRP, Hospital Readmissions Reduction Program; SE, standard error.

at $21.8 \%$ in 2015 , and the proportion of surgery/gynecology hospitalizations in the middle risk stratum (middle three quintiles of risk) decreased from $63.7 \%$ in 2009 to $59.4 \%$ in 2011 to $57.1 \%$ in 2015. Low-risk hospitalizations in the medicine cohort decreased from $21.7 \%$ in 2009 to $19.0 \%$ in 2015 , while high-risk hospitalizations increased from $18.2 \%$ to $20.7 \%$ during the period. Hospitalizations in the lowest stratum of risk steadily declined in both the cardiovascular and neurology cohorts, from $24.9 \%$ to $14.8 \%$ and $22.6 \%$ to $17.3 \%$ of hospitalizations during the period, respectively; this was accompanied by an increase in the proportion of high-risk hospitalizations in each of these cohorts from $16.0 \%$ to $23.4 \%$ and $17.8 \%$ to $21.6 \%$, respectively. The proportion of hospitalizations in each of the 3 risk strata remained relatively stable in the cardiorespiratory cohort (Appendix Table 2).

In each of the five cohorts, O/E readmissions steadily declined from 2009 to 2015 for hospitalizations with the lowest, middle, and highest predicted readmission risk (Figure 2). Each risk stratum had similar rates of decline during the study period for all cohorts (Table 2). Among surgery/gynecology hospitalizations, the monthly O/E readmission declined by 0.030 per year from an initial ratio of 0.936 for the lowest risk hospitalizations, by 0.037 per year for the middle risk hospitalizations, and by 0.036 per year for the highest risk hospitalizations (Table 2). Similarly, for hospitalizations in the lowest versus highest risk of readmission, annual decreases in O/E readmission rates were 0.018 versus $0.015,0.034$ versus $0.033,0.020$ versus 0.015 , and 0.038 versus 0.029 for the medicine, cardiovascular, cardiorespiratory, and neurology cohorts, respectively. For all cohorts and in all risk strata, we found no significant change in $\mathrm{O} / \mathrm{E}$ readmission risk with introduction of ACA or HRRP (Table 2).

\section{DISCUSSION}

In this six-year, national study of Medicare hospitalizations, we found that readmission risk increased over time for surgical and neurological patients but did not increase in medicine or cardiorespiratory hospitalizations, even though those cohorts are known to have had substantial decreases in admissions and readmissions over the same time period. ${ }^{7,8}$ Moreover, we found that $O / E$ readmissions decreased similarly for all hospi- talized Medicare patients, whether of low, moderate, or high risk of readmission. These findings suggest that hospital efforts have resulted in improved outcomes across the risk spectrum.

A number of mechanisms may account for the across-theboard improvements in readmission reduction. Many hospitals have instituted system-wide interventions, including patient education, medicine reconciliation, and early postdischarge follow-up, ${ }^{20}$ which may have reduced readmissions across all patient risk strata. Alternatively, hospitals may have implemented interventions that disproportionally benefited low-risk patients while simultaneously utilizing interventions that only benefited high-risk patients. For instance, increasing threshold for admission ${ }^{7}$ may have the greatest effect on low-risk patients who could be most easily managed at home, while many intensive transitional care interventions have been developed to target only high-risk patients..$^{21,22}$

With the introduction of HRRP, there have been a number of concerns about the readmission measure used to penalize hospitals for high readmission rates. One major concern has been that the readmission metric may be flawed in its ability to capture continued improvement related to readmission. ${ }^{23}$ Some have suggested that with better population health management, admissions will decrease, patient risk of the remaining patients will increase, and hospitals will be increasingly filled with patients who have high likelihood of readmission. This potential for increased risk with HRRP was suggested by a recent study that found that comorbidities increased in hospitalized Medicare beneficiaries between 2010 and 2013. ${ }^{11}$ Our results were mixed in supporting this potential phenomenon because we examined global risk of readmission and found that some of the cohorts had increased risk over time while others did not. Others have expressed concern that readmission measure does not account for socioeconomic status, which has been associated with readmission rates. ${ }^{24-27}$ Although we did not directly examine socioeconomic status in our study, we found that hospitals have been able to reduce readmission across all levels of risk, which includes markers of socioeconomic status, including race and Medicaid eligibility status.

Although we hypothesized that readmission risk would increase as number of hospitalizations decreased over time, we 

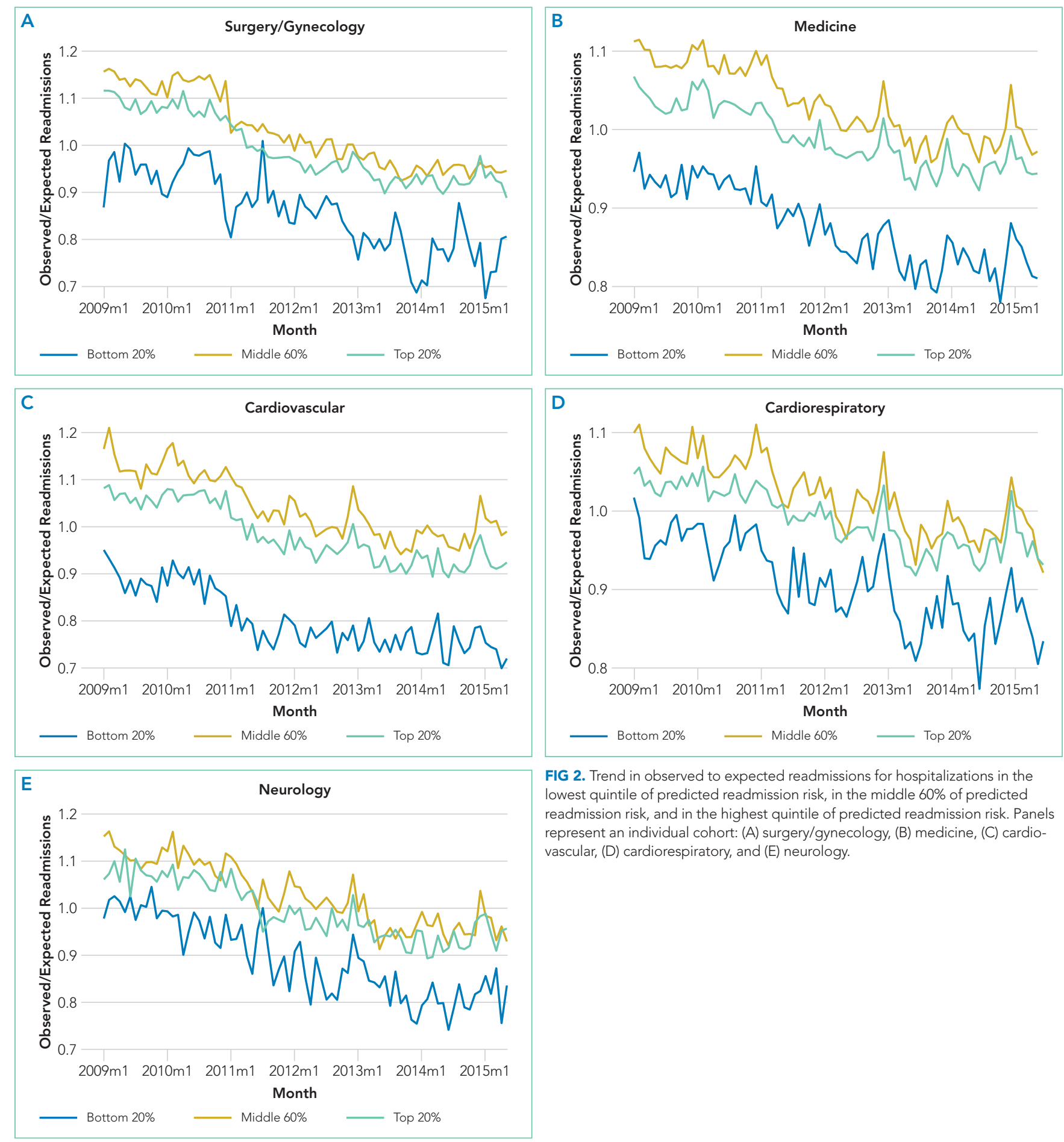

FIG 2. Trend in observed to expected readmissions for hospitalizations in the lowest quintile of predicted readmission risk, in the middle $60 \%$ of predicted readmission risk, and in the highest quintile of predicted readmission risk. Panels represent an individual cohort: (A) surgery/gynecology, (B) medicine, (C) cardiovascular, (D) cardiorespiratory, and (E) neurology.

found no increase in readmission risk among the cohorts with HRRP diagnoses that had the largest decrease in readmission rates. ${ }^{7,8}$ Conversely, readmission risk did increase - with a concurrent increase in the proportion of high-risk hospitalizations - in the surgery/gynecology and neurology cohorts that were not subject to HRRP penalties. Nonetheless, rehospitalizations were reduced for all risk categories in these two cohorts. Notably, surgery/gynecology and neurology had the lowest readmission rates overall. These findings suggest that initiatives to

prevent initial hospitalizations, such as increasing the threshold for postoperative admission, may have had a greater effect on low- versus high-risk patients in low-risk hospitalizations. However, once a patient is hospitalized, multidisciplinary strategies appear to be effective at reducing readmissions for all risk classes in these cohorts.

For the three cohorts in which we observed an increase in readmission risk among hospitalized patients, the risk appeared to increase in early 2011. This time was about 10 months after 
TABLE 2. Beta Coefficients for Time Series Models Examining Observed to Expected Readmission for Hospitalizations in the Lowest $20 \%$, Middle $60 \%$, and Highest $20 \%$ of Predicted Risk for Readmission

\begin{tabular}{|c|c|c|c|c|c|c|}
\hline \multirow[b]{2}{*}{ Cohorts } & \multicolumn{2}{|c|}{ Lowest Risk Quintile } & \multicolumn{2}{|c|}{ Middle $60 \%$ of Risk } & \multicolumn{2}{|c|}{ Highest Risk Quintile } \\
\hline & Coefficient (SE) & $P$ Value & Coefficient (SE) & $P$ Value & Coefficient (SE) & $P$ Value \\
\hline \multicolumn{7}{|c|}{ Surgery/Gynecology Cohort } \\
\hline Time (years) & $-0.030(0.009)$ & .001 & $-0.037(0.010)$ & $<.001$ & $-0.036(0.009)$ & $<.001$ \\
\hline Post-ACA & $0.005(0.022)$ & .835 & $-0.021(0.025)$ & .41 & $0.039(0.020)$ & .05 \\
\hline Post-HRRP & $-0.033(0.029)$ & .261 & $0.006(0.030)$ & .848 & $-0.016(0.027)$ & .559 \\
\hline Intercept & $0.936(0.012)$ & $<.001$ & $1.147(0.025)$ & $<.001$ & $1.083(0.027)$ & $<.001$ \\
\hline \multicolumn{7}{|c|}{ Medicine Cohort } \\
\hline Time (years) & $-0.018(0.005)$ & $<.001$ & $-0.022(0.005)$ & $<.001$ & $-0.015(0.006)$ & .014 \\
\hline Post-ACA & $-0.008(0.015)$ & .567 & $-0.001(0.016)$ & .946 & $-0.020(0.019)$ & .276 \\
\hline Post-HRRP & $-0.017(0.014)$ & .221 & $-0.001(0.017)$ & .971 & $-0.000(0.018)$ & .982 \\
\hline Intercept & $0.949(0.012)$ & $<.001$ & $1.103(0.015)$ & $<.001$ & $1.051(0.011)$ & $<.001$ \\
\hline \multicolumn{7}{|c|}{ Cardiovascular Cohort } \\
\hline Time (years) & $-0.034(0.008)$ & $<.001$ & $-0.029(0.007)$ & $<.001$ & $-0.033(0.008)$ & $<.001$ \\
\hline Post-ACA & $-0.021(0.021)$ & .311 & $-0.005(0.024)$ & .85 & $0.000(0.022)$ & .994 \\
\hline Post-HRRP & $0.032(0.028)$ & .246 & $-0.004(0.022)$ & .857 & $0.014(0.024)$ & .543 \\
\hline Intercept & $0.908(0.016)$ & $<.001$ & $1.146(0.018)$ & $<.001$ & $1.073(0.019)$ & $<.001$ \\
\hline \multicolumn{7}{|c|}{ Cardiopulmonary Cohort } \\
\hline Time (years) & $-0.020(0.006)$ & .001 & $-0.019(0.006)$ & .002 & $-0.014(0.004)$ & .001 \\
\hline Post-ACA & $-0.010(0.019)$ & .59 & $-0.002(0.020)$ & .925 & $-0.004(0.014)$ & .798 \\
\hline Post-HRRP & $-0.003(0.017)$ & .85 & $-0.007(0.017)$ & .686 & $-0.012(0.015)$ & .424 \\
\hline Intercept & $0.985(0.012)$ & $<.001$ & $1.080(0.013)$ & $<.001$ & $1.038(0.012)$ & $<.001$ \\
\hline \multicolumn{7}{|c|}{ Neurology Cohort } \\
\hline Time (years) & $-0.038(0.008)$ & $<.001$ & $-0.032(0.007)$ & $<.001$ & $-0.029(0.007)$ & $<.001$ \\
\hline Post-ACA & $-0.023(0.026)$ & .379 & $0.002(0.017)$ & .89 & $-0.004(0.018)$ & .849 \\
\hline Post-HRRP & $0.019(0.025)$ & .442 & $-0.006(0.022)$ & .791 & $0.004(0.023)$ & .866 \\
\hline Intercept & $1.036(0.019)$ & $<.001$ & $1.134(0.016)$ & $<.001$ & $1.088(0.012)$ & $<.001$ \\
\hline
\end{tabular}

NOTE: Years represents change with each year; post-ACA represents change with introduction of the ACA in March 2010; post-HRRP represents change with introduction of the HRRP in October 2012. Adjusted for calendar quarter. Abbreviations: ACA, Affordable Care Act; HRRP Hospital Readmissions Reduction Program; SE, standard error.

passage of ACA, the timing of which was previously associated with a drop in readmission rates, ${ }^{7,8}$ but well before HRRP went into effect in October 2012. The increase in readmission risk coincided with an increase in the number of diagnostic codes that could be included on a hospital claim to Medicare. ${ }^{17}$ This increase in allowable codes allowed us to capture more diagnoses for some patients, potentially resulting in an increase in apparent predicted risk of readmissions. While we adjusted for this in our predictive models, we may not have fully accounted for differences in risk related to coding change. As a result, some of the observed differences in risk in our study may be attributable to coding differences. More broadly, studies demonstrating the success of HRRP have typically examined risk-adjusted rates of readmission., ${ }^{3,7}$ It is possible that a small portion of the observed reduction in risk-adjusted readmission rates may be related to the increase in predicted risk of readmission observed in our study. Future assessment of trends in readmission during this period should consider accounting for change in the number of allowed billing codes.

Other limitations should be considered in the interpretation of this study. First, like many predictive models for readmission, ${ }^{14}$ ours had imperfect discrimination, which could affect our results. Second, our study was based on older Medicare patients, so findings may not be applicable to younger patients. Third, while we accounted for surrogates for socioeconomic status, including dual eligibility and race, our models lacked other socioeconomic and community factors that can influence readmission. ${ }^{24-26}$ Nonetheless, 1 study suggested that easily measured socioeconomic factors may not have a strong influence on the readmission metric used by Medicare. ${ }^{28}$ Fourth, while our study included over 47 million hospitalizations, our time trend analyses used calendar month as the primary independent variable. As our study included 77 months, we may not have had sufficient power to detect small changes in risk over time.

Medicare readmissions have declined steadily in recent years, presumably at least partly in response to policy changes including HRRP. We found that hospitals have been effective at reducing readmissions across a range of patient risk strata and clinical conditions. As a result, the overall risk of readmission for hospitalized patients has remained constant for some but not all conditions. Whether institutions can continue to reduce readmission rates for most types of patients remains to be seen. 


\section{Acknowledgments}

This study was supported by the Agency for Healthcare Research and Quality (AHRQ) grant R01HS022882. Dr. Blecker was supported by the AHRQ grant K08HS23683. The authors would like to thank Shawn Hoke and Jane Padikkala for administrative support.

Disclosure: This study was supported by the Agency for Healthcare Research and Quality (AHRQ) grants R01HS022882 and K08HS23683. The authors have no conflicts to report.

\section{References}

1. Jha AK. Seeking Rational Approaches to Fixing Hospital Readmissions. JAMA. 2015;314(16):1681-1682.

2. Centers for Medicare \& Medicaid Services. Readmissions Reduction Program. https://www.cms.gov/Medicare/Medicare-Fee-for-Service-Payment/AcutelnpatientPPS/Readmissions-Reduction-Program.html. Accessed on January 17, 2017.

3. Suter LG, Li SX, Grady JN, et al. National patterns of risk-standardized mortality and readmission after hospitalization for acute myocardial infarction, heart failure, and pneumonia: update on publicly reported outcomes measures based on the 2013 release. J Gen Intern Med. 2014:29(10):1333-1340.

4. Gerhardt G, Yemane A, Hickman P, Oelschlaeger A, Rollins E, Brennan N Medicare readmission rates showed meaningful decline in 2012. Medicare Medicaid Res Rev. 2013;3(2):pii:mmrr.003.02.b01.

5. Centers for Medicare and Medicaid Services. New Data Shows Affordable Care Act Reforms Are Leading to Lower Hospital Readmission Rates for Medicare Beneficiaries. http://blog.cms.gov/2013/12/06/new-data-showsaffordable-care-act-reforms-are-leading-to-lower-hospital-readmissionrates-for-medicare-beneficiaries/. Accessed on January 17, 2017.

6. Krumholz HM, Normand SL, Wang Y. Trends in hospitalizations and outcomes for acute cardiovascular disease and stroke, 1999-2011. Circulation. 2014:130(12):966-975.

7. Zuckerman RB, Sheingold SH, Orav EJ, Ruhter J, Epstein AM. Readmissions, Observation, and the Hospital Readmissions Reduction Program. N Engl J Med. 2016;374(16):1543-1551

8. Desai NR, Ross JS, Kwon JY, et al. Association Between Hospital Penalty Status Under the Hospital Readmission Reduction Program and Readmission Rates for Target and Nontarget Conditions. JAMA. 2016;316(24):2647-2656.

9. Brock J, Mitchell J, Irby K, et al. Association between quality improvement for care transitions in communities and rehospitalizations among Medicare beneficiaries. JAMA. 2013;309(4):381-391.

10. Jencks S. Protecting Hospitals That Improve Population Health. http://medicaring.org/2014/12/16/protecting-hospitals/. Accessed on January 5, 2017.

11. Dharmarajan K, Qin L, Lin Z, et al. Declining Admission Rates And Thirty-Day Readmission Rates Positively Associated Even Though Patients Grew Sicker Over Time. Health Aff (Millwood). 2016;35(7):1294-1302.

12. Krumholz HM, Nuti SV, Downing NS, Normand SL, Wang Y. Mortality, Hospitalizations, and Expenditures for the Medicare Population Aged 65 Years or Older, 1999-2013. JAMA. 2015;314(4):355-365.
13. Amarasingham R, Moore BJ, Tabak YP, et al. An automated model to identify heart failure patients at risk for 30-day readmission or death using electronic medical record data. Med Care. 2010;48(11):981-988.

14. Kansagara D, Englander H, Salanitro A, et al. Risk prediction models for hospital readmission: a systematic review. JAMA. 2011;306(15):1688-1698.

15. Horwitz LI, Partovian C, Lin Z, et al. Development and use of an administrative claims measure for profiling hospital-wide performance on 30-day unplanned readmission. Ann Intern Med. 2014;161(10 Suppl):S66-S75.

16. 2016 Condition-Specific Measures Updates and Specifications Report Hospital-Level 30-Day Risk-Standardized Readmission Measures. https://www cms.gov/Medicare/Quality-Initiatives-Patient-Assessment-Instruments/HospitalQualitylnits/Downloads/AMI-HF-PN-COPD-and-Stroke-ReadmissionUpdates.zip. Accessed on January 19, 2017.

17. Centers for Medicare \& Medicaid Services. Pub 100-04 Medicare Claims Processing, Transmittal 2028. https://www.cms.gov/Regulations-and-Guidance/ Guidance/Transmittals/downloads/R2028CP.pdf. Accessed on November 28,2016

18. Martens FK, Tonk EC, Kers JG, Janssens AC. Small improvement in the area under the receiver operating characteristic curve indicated small changes in predicted risks. J Clin Epidemiol. 2016;79:159-164.

19. Blecker S, Goldfeld K, Park H, et al. Impact of an Intervention to Improve Weekend Hospital Care at an Academic Medical Center: An Observational Study. J Gen Intern Med. 2015;30(11):1657-1664.

20. Hansen LO, Young RS, Hinami K, Leung A, Williams MV. Interventions to reduce 30-day rehospitalization: a systematic review. Ann Intern Med. 2011;155(8):520-528

21. Cavanaugh JJ, Jones CD, Embree G, et al. Implementation Science Workshop: primary care-based multidisciplinary readmission prevention program. J Gen Intern Med. 2014;29(5):798-804.

22. Jeng GY, Doyle MM, Belton BM, Herrin J, Horwitz LI. Quasi-Experimental Evaluation of the Effectiveness of a Large-Scale Readmission Reduction Program. JAMA Intern Med. 2016;176(5):681-690.

23. Lynn J, Jencks S. A Dangerous Malfunction in the Measure of Readmission Reduction. http://medicaring.org/2014/08/26/malfunctioning-metrics/. Accessed on January 17, 2017.

24. Calvillo-King L, Arnold D, Eubank KJ, et al. Impact of social factors on risk of readmission or mortality in pneumonia and heart failure: systematic review. $J$ Gen Intern Med. 2013;28(2):269-282.

25. Barnett ML, Hsu J, McWilliams JM. Patient Characteristics and Differences in Hospital Readmission Rates. JAMA Intern Med. 2015;175(11):1803-1812.

26. Singh $S$, Lin $Y L$, Kuo YF, Nattinger $A B$, Goodwin JS. Variation in the risk of readmission among hospitals: the relative contribution of patient, hospital and inpatient provider characteristics. J Gen Intern Med. 2014;29(4):572-578.

27. American Hospital Association. American Hospital Association (AHA) Detailed Comments on the Inpatient Prospective Payment System (PPS) Pro posed Rule for Fiscal Year (FY) 2016. http://www.aha.org/advocacy-issues/ letter/2015/150616-cl-cms1632-p-ipps.pdf. Accessed on January 10, 2017.

28. Bernheim SM, Parzynski CS, Horwitz L, et al. Accounting For Patients' Socioeconomic Status Does Not Change Hospital Readmission Rates. Health Aff (Millwood). 2016:35(8):1461-1470. 